\title{
EXISTENCE OF A POSITIVE SOLUTION FOR AN NTH ORDER BOUNDARY VALUE PROBLEM FOR NONLINEAR DIFFERENCE EQUATIONS
}

\author{
JOHNNY HENDERSON AND SUSAN D. LAUER
}

Abstract. The nth order eigenvalue problem:

$$
\begin{gathered}
\Delta^{n} x(t)=(-1)^{n-k} \lambda f(t, x(t)), \quad t \in[0, T], \\
x(0)=x(1)=\cdots=x(k-1)=x(T+k+1)=\cdots=x(T+n)=0,
\end{gathered}
$$

is considered, where $n \geq 2$ and $k \in\{1,2, \ldots, n-1\}$ are given. Eigenvalues $\lambda$ are determined for $f$ continuous and the case where the limits $f_{0}(t)=$ $\lim _{n \rightarrow 0^{+}} \frac{f(t, u)}{u}$ and $f_{\infty}(t)=\lim _{n \rightarrow \infty} \frac{f(t, u)}{u}$ exist for all $t \in[0, T]$. Guo's fixed point theorem is applied to operators defined on annular regions in a cone.

\section{INTRODUCTION}

Define the operator $\Delta$ to be the forward difference

$$
\Delta u(t)=u(t+1)-u(t),
$$

and then define

$$
\Delta^{i} u(t)=\Delta\left(\Delta^{i-1} u(t)\right), i \geq 1 .
$$

For $a<b$ integers define the discrete interval $[a, b]=\{a, a+1, \ldots, b\}$. Let the integers $n, T \geq 2$ be given, and choose $k \in\{1,2, \ldots, n-1\}$. Consider the nth order nonlinear difference equation

$$
\Delta^{n} x(t)=(-1)^{n-k} \lambda f(t, x(t)), t \in[0, T],
$$

satisfying the boundary conditions

$$
x(0)=x(1)=\cdots=x(k-1)=x(T+k+1)=\cdots=x(T+n)=0 .
$$

1991 Mathematics Subject Classification. 39A10, 34B15.

Key words and phrases. Nth order difference equation, eigenvalue, boundary value problem, fixed point theorem, discrete, nonlinear, Green's function.

Received: October 26, 1997. 
We determine eigenvalues $\lambda$ that yield a solution to (1) and (2), where

$$
\text { (A) } f:[0, T] \times \mathfrak{R}^{+} \rightarrow \mathfrak{R}^{+}
$$

is continuous, where $\mathfrak{R}^{+}$denotes the nonnegative reals,

(B) For all $t \in[0, T], f_{0}(t)=\lim _{u \rightarrow 0^{+}} \frac{f(t, u)}{u}$ and $f_{\infty}(t)=\lim _{n \rightarrow \infty} \frac{f(t, u)}{u}$ both exist.

We apply Guo's fixed point theorem using cone methods, Guo and Lakshmikantham [14], and Krasnosel'skii [19], to accomplish this. This method was first applied to differential equations in the landmark paper by Erbe and Wang [12]. Our proof will follow along the lines of those in Henderson [16], Lauer [17], and Merdivenci [20], additionally utilizing techniques from Peterson [21], Hartman [15], Eloe and Kaufmann [11], Agarwal and Wong [6,7], Agarwal and Henderson [1], and Agarwal, Henderson and Wong [2]. A key to applying this fixed point theorem involves discrete concavity of solutions of the boundary value problem in conjunction with a lower bound on an appropriate Green's function. Extensive use of the results by Eloe [8] concerning a lower bound for the Green's function will be made. Related results for nth order differential equation may be found in Agarwal and Wong $[3,4]$, Eloe and Henderson [9,10], and Fang [13].

\section{Preliminaries}

Let $G(t, s)$ be the Green's funtion for the disconjugate boundary value problem

$$
L x(t) \equiv \Delta^{n} x(t)=0, t \in[0, T],
$$

and satisfying (2), where, as shown in Kelly and Peterson [18], $G(t, s)$ is the unique function satisfying:

(a) $G(t, s)$ is defined for all $t \in[0, T+n], s \in[0, T]$

(b) $L G(t, s)=\delta_{t s}$ for all $t \in[0, T], s \in[0, T]$ where $\delta_{t s}=1$ if $t=s, \delta_{t s}=0$ if $t \neq s$,

(c) For all $s \in[0, T], G(t, s)$ satisfies the boundary conditions (2) in $t$.

We will use $G(t, s)$ as the kernel of an integral operator preserving a cone in a Banach space. This is the setting for our fixed point theorem.

Let $\mathcal{B}$ be a Banach space and let $\mathcal{P} \subset \mathcal{B}$ be such that $\mathcal{P}$ is closed and non-empty. Then $\mathcal{P}$ is a cone provided (i) $a u+b v \in \mathcal{P}$ for all $u, v \in \mathcal{P}$ and for all $a, b \geq 0$, and (ii) $u,-u \in \mathcal{P}$ implies $u=0$.

Applying the following fixed point theorem from Guo, Guo and Lakshmikantham [14], will yield solutions of (1), (2) for certain $\lambda$.

Theorem 1. Let $\mathcal{B}$ be a Banach space, and let $\mathcal{P} \subset \mathcal{B}$ be a cone. Let $\Omega_{1}$ and $\Omega_{2}$ be two bounded open sets in $\mathcal{B}$ such that $0 \in \Omega_{1} \subset \bar{\Omega}_{1} \subset \Omega_{2}$, and let

$$
H: \mathcal{P} \cap\left(\bar{\Omega}_{2} \backslash \Omega_{1}\right) \rightarrow \mathcal{P}
$$

be a completely continuous operator such that, either 
(i) $\|H x\| \leq\|x\|, x \in \mathcal{P} \cap \partial \Omega_{1}$, and $\|H x\| \geq\|x\|, x \in \mathcal{P} \cap \partial \Omega_{2}$, or

(ii) $\|H x\| \geq\|x\|, x \in \mathcal{P} \cap \partial \Omega_{1}$, and $\|H x\| \leq\|x\|, x \in \mathcal{P} \cap \partial \Omega_{2}$.

Then $H$ has a fixed point in $\mathcal{P} \cap\left(\bar{\Omega}_{2} \backslash \Omega_{1}\right)$.

We now apply Theorem 1 to the eigenvalue problem (1), (2), following along the lines of methods incorporated by Henderson [16]. Note that $x(t)$ is a solution of (1), (2) if, and only if,

$$
x(t)=(-1)^{n-k} \lambda \sum_{s=0}^{T} G(t, s) f(s, x(s)), \quad t \in[0, T] .
$$

Hartman [15] extensively studied the boundary value problem (1), (2), with $(-1)^{n-k} \lambda f(t, u) \geq 0$. We begin by stating three Lemmas from Hartman.

Lemma 1. Let $G(t, s)$ denote the Green's function of (3), (2). Then

$$
(-1)^{n-k} G(t, s) \geq 0, \quad(t, s) \in[k, T+k] \times[0, T] .
$$

Lemma 2. Assume that $u$ satisfies the difference inequality $(-1)^{n-k} \Delta^{n} u(t) \geq 0, t \in[0, T]$, and the homogeneous boundary conditions, (2). Then $u(t) \geq 0, t \in[0, T+k]$.

Lemma 3. Suppose that the finite sequence $u(0), \ldots, u(j)$ has $N_{j}$ nodes and the sequence $\Delta u(0), \ldots, \Delta u(j-1)$ has $M_{j}$ nodes. Then $M_{j} \geq N_{j}-1$.

Eloe [8] employed these three lemmas to arrive at the following theorem that gives a lower bound for the solution to the class of boundary value problems studied by Hartman.

Theorem 2. Assume that u satisfies the difference inequality $(-1)^{n-k} \Delta^{n} u(t) \geq 0, t \in[0, T]$, and the homogeneous boundary conditions, (2). Then for $t \in[k, T+k]$,

$$
(-1)^{n-k} u(t) \geq \frac{\nu !}{[(T+1) \cdots(T+\nu)]}\|u\|,
$$

where $\|u\|=\max _{t \in[k, T+k]}|u(t)|$ and $u=\max \{k, n-k\}$.

We remark that Agarwal and Wong [5] have recently sharpened the inequality of Theorem 2. However, this sharper inequality is of little consequence for this work.

Eloe also contributed the following corollary.

Corollary 1. Let $G(t, s)$ denote the Green's function for the boundary value problem, (3), (2). Then for all $s \in[0, T], t \in[k, T+k]$,

$$
(-1)^{n-k} G(t, s) \geq \frac{\nu !}{[(T+1) \cdots(T+\nu)]}\|G(\cdot, s)\|,
$$

where $\|G(\cdot, s)\|=\max _{t \in[k, T+k]}|G(t, s)|$ and $\nu=\max \{k, n-k\}$. 
To fulfill the hypotheses of Theorem 1, let

$$
\begin{aligned}
\mathcal{B}=\{u:[0, T+n] & \rightarrow \mathfrak{R} \quad u(0)=u(1)=\cdots=u(k-1) \\
& =u(T+k+1)=\cdots=u(T+n)=0\},
\end{aligned}
$$

with $\|u\|=\max _{t \in[t, T+k]}|u(t)|$. Now, $(\mathcal{B},\|\cdot\|)$ is a Banach space.

Let

$$
\sigma=\frac{\nu !}{[(T+1) \cdots(T+\nu)]}
$$

and define a cone

$$
\mathcal{P}=\left\{u \in \mathcal{B} \mid u(t) \geq 0 \text { on }[0, T+n] \text { and } \min _{t \in[k, T+k]} u(t) \geq \sigma\|u\|\right\} .
$$

Also choose $\tau, \eta \in[k, T+k]$ such that

$$
\begin{gathered}
(-1)^{n-k} \sum_{s=k}^{T} G(\tau, s) f_{\infty}(s)=\max _{t \in[k, T+k]} \sum_{s=k}^{T} G(t, s) f_{\infty}(s), \\
(-1)^{n-k} \sum_{s=k}^{T} G(\eta, s) f_{0}(s)=\max _{t \in[k, T+k]} .(-1)^{n-k} \sum_{s=k}^{T} G(t, s) f_{0}(s),
\end{gathered}
$$

\section{Main Results}

Theorem 3. Assume conditions (A) and (B) are satisfied. Then, for each $\lambda$ satisfying

$$
\frac{1}{\sigma(-1)^{n-k} \sum_{s=0}^{T} G(\tau, s) f_{\infty}(s)}<\lambda<\frac{1}{\sum_{s=k}^{T}\|G(\cdot, s)\| f_{0}(s)}
$$

there exists at least one solution of (1), (2) in $\mathcal{P}$.

Proof. Let $\lambda$ be given as in Theorem 3. Let $\epsilon>0$ be such that

$$
\frac{1}{\sigma(-1)^{n-k} \sum_{s=k}^{T} G(\tau, s)\left(f_{\infty}(s)-\epsilon\right)} \geq \lambda \geq \frac{1}{\sum_{s=0}^{T}\|G(\cdot, s)\|\left(f_{0}(s)+\epsilon\right)} .
$$

Define a summation operator $H: \mathcal{P} \rightarrow \mathcal{B}$ by

$$
H x(t)=(-1)^{n-k} \lambda \sum_{s=0}^{T} G(t, s) f(s, x(s)), \quad x \in \mathcal{P} .
$$

We seek a fixed point of $H$ in the cone $\mathcal{P}$. By the nonnegativity of $f$ and $(-1)^{n-k} G, H x(t) \geq 0$ on $[0, T+n]$, and from the properties of $G, H x$ 
satisfies the boundary conditions. Now if we choose $x \in \mathcal{P}$, we have

$$
\begin{aligned}
H x(t) & =(-1)^{n-k} \lambda \sum_{s=0}^{T} G(t, s) f(s, x(s)) \\
& \leq \lambda \sum_{0=0}^{T}\|G(\cdot, s)\| f(s, x(s)), t \in[k, T+k] .
\end{aligned}
$$

So

$$
\|H x\|=\max _{t \in[k, T+k]}|H x(t)| \leq \lambda \sum_{s=0}^{T}\|G(\cdot, s)\| f(s, x(s)) .
$$

Hence, if $x \in \mathcal{P},(-1)^{n-k} G(t, s) \geq \sigma\|G(\cdot, s)\|$, for $t \in[k, T+k]$ and $s \in[0, T]$, and thus,

$$
\begin{aligned}
& \min _{t \in[k, T+k]} H x(t)=\min _{k, T+k]}(-1)^{n-k} \lambda \sum_{s=0}^{T} G(t, s) f(s, x(s)) \\
& \geq \sigma \lambda \sum_{s=0}^{T}\|G(\cdot, s)\| f(s, x(s)) \\
& \geq \sigma\|H x\| .
\end{aligned}
$$

Thus $H: \mathcal{P} \rightarrow \mathcal{P}$. Additionally, $H$ is completely continuous.

Now consider $f_{0}(t)$. For each $t \in[0, T]$, there exists $k_{t}>0$ such that $f(t, u) \leq\left(f_{0}(t)+\epsilon\right) u$ for $0<u \leq k_{t}$. Let $K_{1}=\min _{t \in[0, T]} k_{t}$. So, for $x \in \mathcal{P}$ with $\|x\|=K_{1}$, we have

$$
\begin{aligned}
H x(t) & =(-1)^{n-k} \lambda \sum_{s=0}^{T} G(t, s) f(s, x(s)) \\
& \leq \lambda \sum_{s=0}^{T}\|G(\cdot, s)\|\left(f_{0}(s)+\epsilon\right) x(s) \\
& \leq \lambda \sum_{s=0}^{T}\|G(\cdot, s)\|\left(f_{0}(s)+\epsilon\right)\|x\| \\
& \leq\|x\|, \quad t \in[k, T+k] .
\end{aligned}
$$

Therefore, $\|H(x)\| \leq\|x\|$. Hence, if we set

$$
\Omega_{1}=\left\{u \in \mathcal{B} \mid\|u\|<K_{1}\right\}
$$

then

$$
\|H x\| \leq\|x\| \text { for all } x \in \mathcal{P} \cap \partial \Omega_{1} .
$$

Next consider $f_{\infty}(t)$. For each $t \in[0, T]$, there exists $\tilde{k}_{t}>0$ such that $f(t, u) \geq\left(f_{\infty}(t)-\epsilon\right) u$ for all $u \geq \tilde{k}_{t}$. Let $\tilde{K}_{2}=\max _{t \in[0, T]} \tilde{k}_{t}$ and $K_{2}=$ 
$\max \left\{2 K_{1}, \frac{1}{\sigma} \tilde{K}_{2}\right\}$. Define

$$
\Omega_{2}=\left\{u \in \mathcal{B} \mid\|u\|<K_{2}\right\}
$$

If $x \in \mathcal{P}$ with $\|x\|=K_{2}$, then $\min _{t \in[k, T+k]} x(t) \geq \sigma\|x\| \geq \tilde{K}_{2}$, and

$$
\begin{aligned}
H x(\tau) & =(-1)^{n-k} \lambda \sum_{s=0}^{T} G(\tau, s) f(s, x(s)) \\
& \leq(-1)^{n-k} \lambda \sum_{s=0}^{T} G(\tau, s) f(s, x(s)) \\
& \left.\geq(-1)^{n-k} \lambda \sum_{s=0}^{T} G(\tau, s) f_{\infty}(s)-\epsilon\right) x(s)(s, x(s)) \\
& \geq \sigma(-1)^{n-k} \lambda \sum_{s=k}^{T} G(\tau, s)\left(f_{\infty}(s)-\epsilon\right)\|x\| \\
& \geq\|x\| .
\end{aligned}
$$

Thus, $\|H x\| \geq\|x\|$, and so

$$
\|H x\| \geq\|x\| \text { for all } x \in \mathcal{P} \cap \partial \Omega_{2}
$$

So with (8) and (9) we have shown that $H$ satisfies the first condition of Theorem 1. Thus we can conclude that $H$ has a fixed point $u(t) \in \mathcal{P} \cap$ $\left(\bar{\Omega}_{2} \backslash \Omega_{1}\right)$. This fixed point, $u(t)$, is a solution of $(1),(2)$ corresponding to the given value of $\lambda$.

Theorem 4. Assume conditions (A) and (B) are satisfied. Then, for each $\lambda$ satisfying

$$
\frac{1}{\sigma(-1)^{n-k} \sum_{s=k}^{T} G(\eta, s) f_{0}(s)}<\lambda<\frac{1}{\sum_{s=0}^{T}\|G(\cdot, s)\| f_{\infty}(s)},
$$

there exists at least solution of (1), (2) in $\mathcal{P}$.

Proof. Let $\lambda$ be given as stated above. Let $\epsilon>0$ be such that

$$
\frac{1}{\sigma(-1)^{n-k} \sum_{s=k}^{T} G(\eta, s)\left(f_{0}(s)-\epsilon\right)} \leq \lambda \leq \frac{1}{\sum_{s=0}^{T}\|G(\cdot, s)\|\left(f_{\infty}(s)+\epsilon\right)}
$$

Let $H$ be the cone preserving, completely continuous operator defined in (7).

Consider $f_{0}(t)$. For each $t \in[0, T]$ there exists $k_{t}>0$ such that $f(t, u) \geq$ $\left(f_{0}(t)-\epsilon\right) u$ for $0<u \leq k_{t}$. Let $K_{1}=\min _{t \in[0, T]} k_{t}$. So, for $x \in \mathcal{P}$ with $\|x\|=K_{1}$, 
we have

$$
\begin{aligned}
H x(\eta) & =(-1)^{n-k} \lambda \sum_{s=0}^{T} G(\eta, s) f(s, x(s)) \\
& \geq(-1)^{n-k} \lambda \sum_{s=k}^{T} G(n, x) f(x, x(s)) \\
& \geq(-1)^{n-k} \lambda \sum_{s=0}^{T} G(\eta, s)\left(f_{0}(s)-\epsilon\right) x(s) \\
& \geq \sigma(-1)^{n-k} \lambda \sum_{s=k}^{T} G(\eta, s)\left(f_{0}(s)-\epsilon\right)\|x\| \\
& \geq\|x\| .
\end{aligned}
$$

Therefore, $\|H x\| \geq\|x\|$. Hence, if we set

$$
\Omega_{1}=\left\{u \in \mathcal{B} \mid\|u\|<K_{1}\right\},
$$

$$
\|H x\| \geq\|x\|, \text { for all } x \in \mathcal{P} \cap \partial \Omega_{1} .
$$

Next consider $f_{\infty}(t)$. For each $t \in[0, T]$ there exists $\tilde{k}_{t}>2 K_{1}$ such that $f(t, u) \leq\left(f_{\infty}(t)+\epsilon\right) u$ for all $u \geq \tilde{k_{t}}$. There exists sets $I, J \subset[0, T]$, with $I \cup J=[0, T]$, such that for all $t \in I, f(t, u)$ is bounded as a function of $u$, and for all $t \in J, f(t, u)$ is unbounded as a function of $u$.

Choose $M>0$ such that for all positive $u$ and for all $t \in I, f(t, u) \leq M$. Let

$$
\kappa_{t}=\max \left\{\tilde{k}_{t}, \frac{M}{f_{\infty}(t)+\epsilon}\right\}
$$

For each $t \in J$ choose $\kappa_{t} \geq \tilde{k}_{t}$ such that $f(t, u) \leq f\left(t, \kappa_{t}\right)$, for $0<u \leq \kappa_{t}$. Let $K_{2}=\max _{t \in[0, T]} \kappa_{t}$. By the continuity of $f$, for all $t \in J$ there exists $\mu_{t}$, where $\kappa_{t} \leq \mu_{t} \leq K_{2}$, such that $f(t, u) \leq f\left(t, \mu_{t}\right)$ for all $0<u \leq K_{2}$. Now

$$
\begin{aligned}
H x(t) & =(-1)^{n-k} \lambda \sum_{s=0}^{T} G(t, s) f(s, x(s)) \\
& \leq \lambda \sum_{s \in J}\|G(\cdot, s)\| M+\lambda \sum_{s \in I}\|G(\cdot, s)\| f\left(s, \mu_{s}\right) \\
& \leq \lambda \sum_{s \in I}\|G(\cdot, s)\|\left(f_{\infty}(s)+\epsilon\right) \kappa_{s}+\lambda \sum_{s \in J}\|G(\cdot, s)\|\left(f_{\infty}(s)+\epsilon\right) \mu_{s} \\
& \leq \lambda \sum_{s=0}^{T}\|G(\cdot, s)\|\left(f_{\infty}(s)+\epsilon\right) K_{2} \\
& =\lambda \sum_{s=0}^{T}\|G(\cdot, s)\|\left(f_{\infty}(s)+\epsilon\right)\|x\| \\
& \leq\|x\|
\end{aligned}
$$


for $x \in \mathcal{P}$ with $\|x\|=K_{2}$. Now if we take

$$
\Omega_{2}=\left\{u \in \mathcal{B} \mid\|u\|<K_{2}\right\},
$$

then

$$
\|H x\| \leq\|x\| \text { for all } x \in \mathcal{P} \cup \partial \Omega_{2} .
$$

Thus, with (10) and (11), we have shown that $H$ satisfies the hypotheses to Theorem 1(ii), which yields a fixed point of $H$ belonging to $\mathcal{P} \cap\left(\bar{\Omega}_{2} \backslash \Omega_{1}\right)$. this fixed point is a solution of (1), (2) corresponding to the given $\lambda$.

\section{REFERENCES}

[1] R. P. Agarwal and J. L. Henderson, Positive solutions and nonlinear eigenvalue problems for third order difference equations, Comput. Math. Appl. (to appear).

[2] R. P. Agarwal, J. L. Henderson, and P. J. Y. Wong, On superlinear and sublinear $(n, p)$ boundary value problems, Nonlinear World (in press).

[3] R. P. Agarwal and P. J. Y. Wong, Eigenvalue characterization for $(n, p)$ boundary value problems, Journal Austral. Math. Soc. Ser. B, (to appear).

[4] R. P. Agarwal and P. J. Y. Wong, Eigenvalues of boundary value problems for higher order differential equations, Math. Probl. Engineering, (to appear).

[5] R. P. Agarwal and P. J. Y. Wong, Extension of continuous and discrete inequalities due to Eloe and Henderson, Nonlinear Anal. (to appear).

[6] R. P. Agarwal and P. J. Y. Wong, On the existence of positive solutions of higher order difference equations, Topol. Methods Nonlinear Anal. (to appear).

[7] R. P. Agarwal and P. J. Y. Wong, On the existence of solutions of singular boundary value problems for higher order difference equations, Nonlinear Anal. 28 (1997), 277-287.

[8] P. W. Eloe, A generalization of concavity for finite differences, Comput. Math. Appl. (to appear).

[9] P. W. Eloe and J. L. Henderson, Positive solutions and nonlinear multipoint conjugate eigenvalue problems, Electron. J. Differential Equations, 3 (1997), 1-11.

[10] P. W. Eloe and J. L. Henderson, Positive solutions for higher order ordinary differential equations, Electron. J. Differential Equations, 3 (1995) 1-8.

[11] P. W. Eloe and E. R. Kaufmann, A unique limiting Green's function for a class of singular boundary value problems, Comput. Math. Appl. 28 (1994), 93-99.

[12] L. H. Erbe and H. Wang, On the existence of positive solutions of ordinary differential equations, Proc. Amer. Math. Soc. 120 (1994), 743-748.

[13] F. Fang, Positive Solutions of a Class of Boundary Value Problems, Ph.D. Dissert., Auburn University, 1997.

[14] D. Guo and V. Lakshmikantham, Nonlinear Problems in Abstract Cones, Academic Press, San Diego, 1988.

[15] P. Hartman, Difference equations: Disconjugacy, principal solutions, Green's functions, complete monotonicity, Trans. Amer. Math. Soc. 2465 (1978), 1-30.

[16] J. L. Henderson, Positive solutions for nonlinear difference equations, Nonlinear Stud. (to appear).

[17] S. D. Lauer, Positive Solutions for nonlinear difference equations, etc. Comm. Appl. Nonlinear Anal. 4, (1997), Number 3.

[18] W. G. Kelley and A. C. Peterson, Difference Equations, An Introduction with Applications, Academic Press, San Diego, 1991.

[19] M. A. Krasnosel'skii, Positive Solutions of Operator Equations, Noordhoff, Groningen, The Netherlands, 1964.

[20] F. Merdivenci, Two positive solutions of a boundary value problem for difference equations, J. Differ. Equations Appl. 1 (1995), 263-270. 
[21] A. C. Peterson, Boundary value problems for an nth order linear difference equation, SIAM J. Math. Anal. 15 (1984), 124-132.

JOHNNY HENDERSON

Department of Mathematics

Auburn University

Auburn, Alabama 38649, USA

E-mail address: hendej2@mail.auburn.edu

Susan D. LAUER

Department of Mathematics

TUSKEgEe UNIVERsity

Tuskegee, Alabama 36088, USA

E-mail address: lauersd@auburn.campus.mci.net 


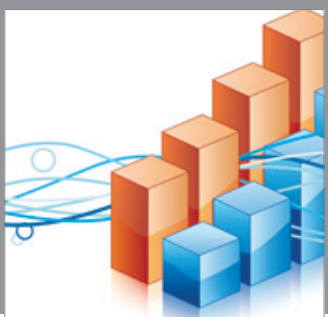

Advances in

Operations Research

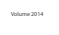

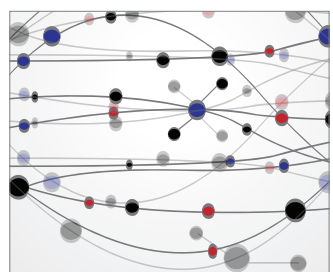

\section{The Scientific} World Journal
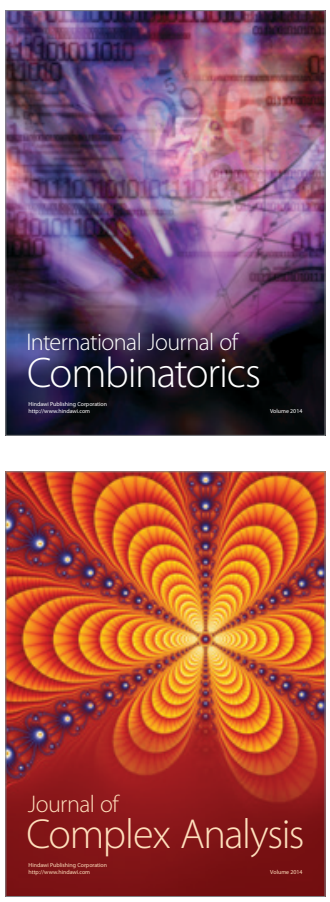

International Journal of

Mathematics and

Mathematical

Sciences
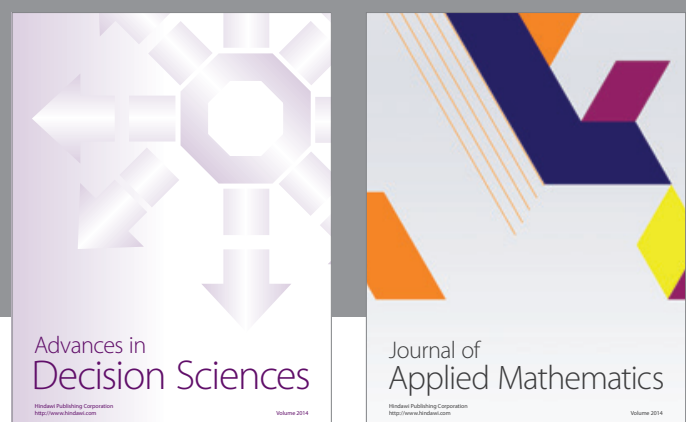

Journal of

Applied Mathematics
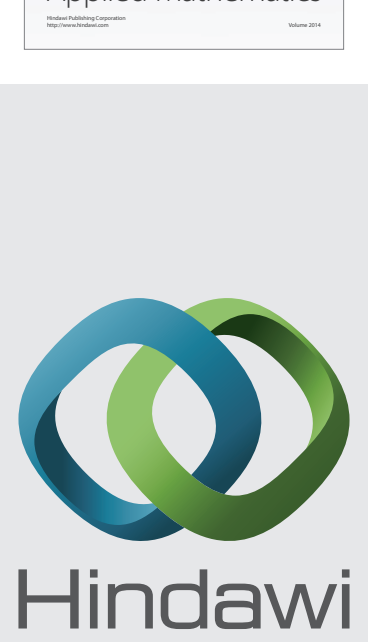

Submit your manuscripts at http://www.hindawi.com
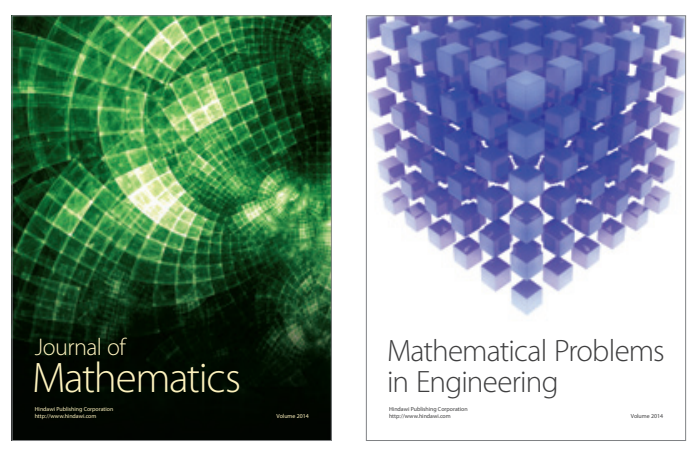

Mathematical Problems in Engineering
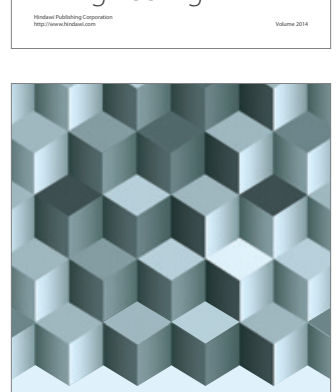

Journal of

Function Spaces
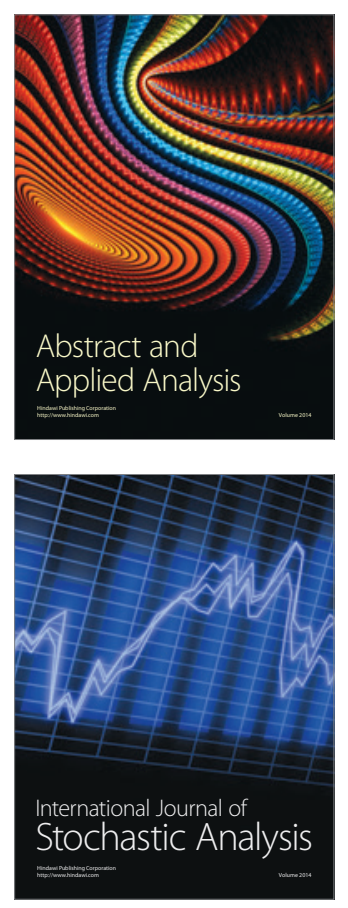

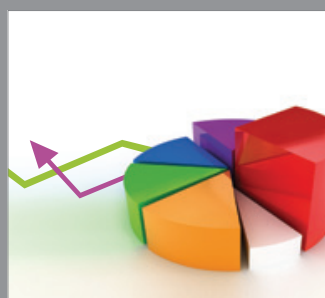

ournal of

Probability and Statistics

Promensencen
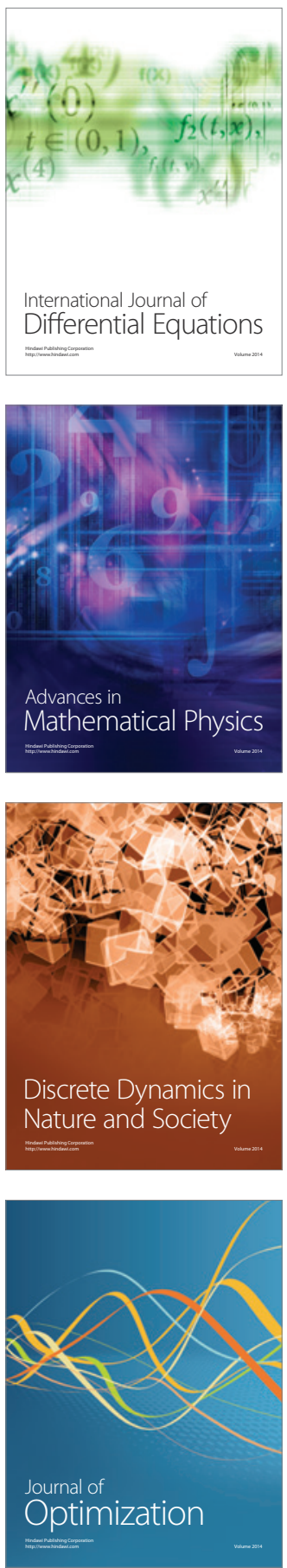